\title{
Effects of Sirt3-autophagy and resveratrol activation on myocardial hypertrophy and energy metabolism
}

\author{
HAI-NING WANG, JI-LIN LI, TAN XU, HUAI-QI YAO, GUI-HUA CHEN and JING HU \\ The First Affiliated Hospital of Shantou University Medical College Cardiac Care Unit, \\ Shantou, Guangdong 515041, P.R. China
}

Received May 9, 2017; Accepted August 10, 2018

DOI: $10.3892 / \mathrm{mmr} .2020 .11195$

\begin{abstract}
The aim of the present study was to examine the role of sirtuin 3 (Sirt3)-autophagy in regulating myocardial energy metabolism and inhibiting myocardial hypertrophy in angiotensin (Ang) II-induced myocardial cell hypertrophy. The primary cultured myocardial cells of neonatal Sprague Dawley rats were used to construct a myocardial hypertrophy model induced with Ang II. Following the activation of Sirt3 by resveratrol (Res), Sirt3 was silenced using small interfering (si)RNA-Sirt3, and the morphology of the myocardial cells was observed under an optical microscope. Reverse transcription-polymerase chain reaction was used to detect the mRNA expression of the following myocardial hypertrophy markers; atrial natriuretic peptide (ANP), brain natriuretic peptide (BNP), Sirt3, medium-chain acyl-CoA dehydrogenase (MCAD) and pyruvate kinase (PK). Western blot analysis was used to detect the protein expression of Sirt3, light chain 3 (LC3) and Beclin1. Ang II may inhibit the protein expression of Sirt3, LC3 and Beclin1. Res, an agonist of Sirt3, may promote the protein expression of Sirt3, LC3 and Beclin1. Res inhibited the mRNA expression of ANP and BNP, and reversed the Ang II-induced myocardial cell hypertrophy. The addition of siRNA-Sirt3 decreased the protein expression of Sirt3, LC3 and Beclin1, increased the mRNA expression of ANP and BNP, and weakened the inhibitory effect of Res on myocardial cell hypertrophy. Res promoted the mRNA expression of MCAD, inhibited the mRNA expression of PK, and reversed the influence of Ang II on myocardial energy metabolism. siRNA-Sirt3 intervention significantly decreased the effect of Res in eliminating abnormal myocardial energy metabolism. In conclusion, Sirt3 may inhibit Ang II-induced myocardial hypertrophy and reverse the Ang II-caused
\end{abstract}

Correspondence to: Dr Hai-Ning Wang, The First Affiliated Hospital of Shantou University Medical College Cardiac Care Unit, 57 Changping Road, Shantou, Guangdong 515041, P. R. China E-mail:wanghnvip@163.com

Key words: resveratrol, myocardiocytes, myocardial hypertrophy, sirtuin 3, autophagy, angiotensin II abnormal myocardial energy metabolism through activation of autophagy.

\section{Introduction}

Myocardial hypertrophy is an independent risk factor of cardiovascular diseases (1). At the molecular level, myocardial hypertrophy is caused by an imbalance of protein synthesis and degradation. Persistent external stimuli lead to overexpression of the signaling pathway for myocardial protein synthesis and inhibition of the metabolic pathway (2). Other effects include increased myocardial cell volume, increased protein synthesis and muscle fibre and myocardial remodeling (3). Furthermore, abnormal energy metabolism, which turns from the oxygenolysis of fatty acids to the utilization of glucose, is additionally accompanied (4). Such a long-term alteration will lead to the reduction of energy production efficiency, the accumulation of fatty acids in myocardial cells, and the increase of the anaerobic glycolysis of glucose, which reduces the myocardial cell contraction and accelerates the occurrence and development of heart failure (5-7). The present study examined the regulation of myocardial cell energy metabolism and the autophagy signaling pathway to determine a mechanism that may inhibit myocardial hypertrophy and may be used as a novel target of treatment.

The primary regulatory factors in cell energy metabolism are AMP-activated protein kinase (AMPK) and members of the silent information regulator family $(7,8)$. Sirtuin3 (Sirt3) belongs to the latter and is a histone deacetylase (HDAC) III (8). As a nicotinamide adenine dinucleotide (NAD)-dependent HDAC primarily existing in the mitochondria, Sirt3 not only regulates the energy metabolism of cells; however, additionally serves an important role in apoptosis, tumor growth and cardiovascular diseases (9). Previously, an increasing number of studies have reported that Sirt3 serves a key role in myocardial hypertrophy. Sundaresan et al (10) observed that Sirt3 may downregulate mitogen-activated protein kinases/extracellular signal-regulated kinases and the phosphoinositide 3-kinase/protein kinase B signaling pathways through inhibition of the oxygen radical-mediated renin activity. This inhibition occurs by activating forkhead box protein $\mathrm{O} 3$ (FoxO3), manganese superoxide dismutase and catalase, and by inhibiting myocardial hypertrophy (10). Pillai et al (11) determined that myocardial hypertrophy may be inhibited through activation of the Sirt3-liver 
kinase B1 (LKB1)-AMPK pathway (11). It was observed that Sirt3 gene knockout mice demonstrated myocardial hypertrophy (12). All of these studies suggest that Sirt3 is involved in the occurrence and development of myocardial hypertrophy.

Autophagy is a biological phenomenon widely available in eukaryocytes. Autophagy is additionally an important channel of waste elimination, structure reconstruction, and growth and development of cells (13). Reduced autophagy has been identified in myocardial hypertrophy since the early 1980s; Dämmrich and Pfeifer (14) identified decreased autophagy in an aortic coarctation model. Nakai et al (15) observed significantly decreased autophagy in a myocardial hypertrophy model induced by aortic coarctation. A previous study published in 2014 (16) additionally demonstrated that the autophagy level decreased significantly in an in vitro myocardial hypertrophy model induced by angiotensin (Ang) II, which suggests that autophagy may inhibit the development of myocardial hypertrophy.

At present, the Sirt3-autophagy pathway is more frequently reported, including in hepatic diseases (17-19), the nervous system $(20,21)$, tumors $(22,23)$ and skeletal muscle (24). In myocardial ischemia reperfusion, Sirt 3 may protect the heart by promoting autophagy (25). However, there is no study at present, to the best of the authors' knowledge, on the Sirt3-autophagy pathway in myocardial hypertrophy.

\section{Materials and methods}

Experimental animals. In total, 12 clean Sprague Dawley rats ( 6 male and 6 female) born within 1-2 days were provided by the Experimental Animal Centre of Shantou University Medical College (Shantou, China) from the same race and brood. The temperature was maintained at $25^{\circ} \mathrm{C}$ and the humidity at was maintained at $50 \%$ with 12 -h light/dark cycle. All the rats had free access food and water. Differences pertaining to a comparison among age, weight (average 5-6 g) and health state were not statistically significant $(\mathrm{P}>0.05)$. The present study was approved by the Medical Ethics Committee of Shantou University.

Primary reagents. Dulbecco's modified Eagle's medium (DMEM)-F12 and fetal calf serum (FCS; HyClone; GE Healthcare Life Sciences), trypsin and collagenase I (Gibco; Thermo Fisher Scientific, Inc.), BrdU (Sigma-Aldrich; Merck KGaA), Ang II (AnaSpec), antibodies against Sirt3 [Cell Signaling Technology (CST), Inc.; C73E3 Rabbit mAb; cat. no. 2627S], light chain (LC)3I/II (CST, Inc.; D11, XP Rabbit mAb; cat. no. 3868S), Beclin1 (CST, Inc.; D40C5, Rabbit mAb; cat. no. 3495S), GADPH (CST, Inc.; D16H11, XP Rabbit mAb; cat. no. 5174S) and $\beta$-actin (CST, Inc.; 13E5, Rabbit mAb; cat. no. 4970T), goat anti-rabbit immunoglobulin G-horseradish peroxidase (HRP) secondary antibody (cat. no. R11412; Bellancom), a western blotting kit (EMD Millipore), nuclease-free water, RNAase inhibitor, deoxyribonucleotide mixture, reverse transcriptase and random primers (Takara Bio, Inc.), and resveratrol (Shanghai Sangon Pharmaceutical Co., Ltd.) were used.

Separation and culture of primary myocardial cells of neonatal rats. The heart was removed by thoracotomy under aseptic conditions and submerged in D-Hank's solution. Subsequent to the bloodiness being cleaned, the connective tissue of the cardiac base and the atrial tissue were removed. The ventricle was cut off to remove the extravasated blood. The myocardial tissue was placed into a $15 \mathrm{ml}$ centrifuge tube and cut into blocks of $1 \mathrm{~mm}^{3}$. After $0.5 \mathrm{~g} / \mathrm{l}$ collagenase type I was added, the tube was gently oscillated in a $37^{\circ} \mathrm{C}$ thermostatic bath for $2 \mathrm{~h}$ to lyse the tissue block. Subsequently, $0.125 \mathrm{~g} / 1$ pancreatin was added to digest the tissue and this was conducted 2-3 times ( $5 \mathrm{~min} / \mathrm{time})$. The digested cells were collected with medium containing $100 \mathrm{ml} / 1$ FCS, inoculated into a culture dish, and placed into a $5 \% \mathrm{CO}_{2}$ incubator for $1 \mathrm{~h}$ at $37^{\circ} \mathrm{C}$. Fibroblasts were eliminated by differential adhesion. Myocardial cells were inoculated into a 6 -well plate at a density of $1 \times 10^{6} / \mathrm{ml}$. A total of $0.03 \mathrm{~g} / 1 \mathrm{BrdU}$ was added to inhibit fibroblast growth. A total of $0.1 \mathrm{~g} / \mathrm{l}$ penicillin and $0.1 \mathrm{~g} / \mathrm{l}$ streptomycin were added to prevent bacterial contamination. Subsequently, the plate was cultured in a $50 \mathrm{ml} / \mathrm{C} \mathrm{CO}_{2}$ incubator. At $24 \mathrm{~h}$ after inoculation and when the cells fused and contracted synchronously, the serum-free medium was used. After $24 \mathrm{~h}$, intervention of the different groups was conducted (26).

Protein expression is detected by western blot analysis. Following the completion of the aforementioned treatment, myocardial cells were lysed and the total protein was extracted using SDS buffer (Jianglai Bio, Inc.). A total of $30 \mu \mathrm{l}$ of protein, determined using bicinchoninic acid method, was loaded in each lane of a $12 \%$ of SDS-PAGE gel for electrophoresis. Subsequent to electrophoresis, the protein was transferred to the polyvinylidene difluoride (PVDF) membranes (80 V; $120 \mathrm{~min}$ ); after $1 \mathrm{~h}$ of blocking at $25^{\circ} \mathrm{C}$ using $5 \%$ bovine serum albumin (Gibco; Thermo Fisher Scientific, Inc.), the protein was incubated with the following antibodies overnight at $4^{\circ} \mathrm{C}$; Sirt3 (1:1,000), LC3I/II (1:1,000), Beclin1 (1:1,000), medium-chain acyl-CoA dehydrogenase (MCAD; 1:1,000), GADPH (1:20,000) and $\beta$-actin (1:10,000). Subsequent to washing with Tris-buffered saline with $15 \mathrm{ml}$ of $1 \mathrm{X}$ Tween-20 the following day, the PVDF membranes were incubated with HRP-labelled goat anti-rabbit $(1: 10,000)$ antibody for $1 \mathrm{~h}$ at room temperature. Western blot analysis was conducted using enhanced chemiluminescence substrate kit (Shanghai Yaxin Biotechnology Co., Ltd.). Quantity One image analysis software (version 4.5; Bio-Rad Laboratories, Inc.) was used to detect the grey value of the protein bands. The grey value ratio of the target band and GAPDH or $\beta$-actin was used to indicate the expression level of the target protein (27).

Reverse transcription quantitative polymerase chain reaction $(R T-q P C R)$. The mRNA was extracted by routine TRIzol ${ }^{\circledR}$ lysis (Thermo Fisher Scientific, Inc.) and reverse transcribed into cDNA. Reverse transcription was performed using a PrimeScript RT Reagent kit (Takara Bio, Inc.). GAPDH was used as an internal control. The $\mathrm{PCR}$ reaction conditions were as follows: Initial denaturation at $42^{\circ} \mathrm{C}$ for 60 min followed by $99^{\circ} \mathrm{C}$ for $5 \mathrm{~min}$ and $4^{\circ} \mathrm{C}$ for $5 \mathrm{~min}$. Relative expression of the target gene was calculated using the $2^{-\Delta \Delta \mathrm{Cq}}$ method (28): $\Delta \Delta \mathrm{Cq}=\mathrm{Cq}$ target gene - $\mathrm{Cq}$ reference gene (experimental group), $\mathrm{Cq}$ target gene - $\mathrm{Cq}$ reference gene (control group). $\mathrm{Cq}$ was the number of quantification cycles at which the fluorescence exceeded the threshold. Each experiment was performed in triplicate.

According to the standard curve: i) Using cDNA of the control group as the template, the cDNA was diluted to $1: 10$, 1:50, 1:1,00 and 1:1,000; ii) ligand system: The total volume 
Table I. Primer sequences for the polymerase chain reaction assays.

\begin{tabular}{lcl}
\hline Gene & Primer & \multicolumn{1}{c}{ Primer sequences } \\
\hline Sirt3 & F & 5'-TGCACGGTCTGTCGAAGGTC-3' \\
& R & 5'-AGGTTTCACAACGCCAGTA-3' \\
ANP & F & 5'-CGTATACAGTGCGGTGTCCA-3' \\
& R & 5'-GATCTATCGGAGGGGTCCCA-3' \\
BNP & F & 5'-TCCTTAATCTGTCGCCGCTG-3' \\
& R & 5'-CGCCGATCCGGTCTATCTTC-3' \\
\multirow{2}{*}{ MCAD } & F & 5'-AGCCCTGGACGAAGCTACTA-3' \\
& R & 5'-GCGAGCTGGTTGGCAATATC-3' \\
GAPDH & F & 5'-TGCCACTCAGAAGACTGTGG-3' \\
& R & 5'-TTCAGCTCTGGGATGACCTT-3' \\
\multirow{2}{*}{ B-actin } & F & 5'-GAACCCTAAGGCCAACCGTGAAAAGAT-3' \\
& R & 5'-ACCGCTCGTTGCCAATAGTGATG-3' \\
PK & F & 5'-AATCCCGGCAGATACAGACT-3' \\
& R & 5'-GGAGTTCCACACCCTGCTAT-3' \\
\hline
\end{tabular}

Sirt3, sirtuin 3; ANP, atrial natriuretic peptide; BNP, brain natriuretic peptide; MCAD, medium-chain acyl-CoA dehydrogenase; PK, pyruvate kinase; F, forward; $\mathrm{R}$, reverse.

was $10 \mu \mathrm{l}$ and each sample had three wells $(4 \mu \mathrm{cDNA}+0.3 \mu \mathrm{l}$ Primer $\mathrm{F}+0.3 \mu 1$ Primer $\mathrm{R}+0.4 \mu 1 \mathrm{H}_{2} \mathrm{O}+5 \mu \mathrm{l}$ SYBR-Green (Takara Bio, Inc.) x number of wells; iii) centrifugation: $999 \mathrm{x}$ for $4 \mathrm{~min}$ at $4^{\circ} \mathrm{C}$; and iv) real-time PCR analysis.

The quantitative $\mathrm{PCR}$ was performed according to a previous study (28). First, the cDNA solution was diluted 50 times. Subsequently, the samples $(4 \mu \mathrm{l})$ were loaded. The rest was the same as that above. Samples were run in triplicate. The system was put into the Roche PCR analyzer (Roche Molecular Diagnostics). The reaction conditions were pre-denaturation at $95^{\circ} \mathrm{C}$ for $5 \mathrm{~min}$ followed by processing at $95^{\circ} \mathrm{C}$ for $10 \mathrm{sec}, 60^{\circ} \mathrm{C}$ for $10 \mathrm{sec}$ and $72^{\circ} \mathrm{C}$ for $20 \mathrm{sec}$ for a total of 45 cycles. Following completion of the reaction, DataAssist ${ }^{\mathrm{TM}}$ software (v3.01) (Thermo Fisher Scientific, Inc.) was used for analysis. The analysis was repeated three times. The data were used for statistical analysis (Table I).

Area of myocardial cells. Following treatment with Ang II $(20 \mu \mathrm{M})$ for $48 \mathrm{~h}$ at $37^{\circ} \mathrm{C}$, the myocardial cells were fixed with $4 \%$ paraformaldehyde at $4^{\circ} \mathrm{C}$ for $30 \mathrm{~min}$ and imaged using a fluorescent microscope, magnification, x400. Fifty views of the same size were taken for each group. The image analysis software, Image pro plus 6.0 (National Institutes of Health), was used to calculate the area of the cells (27).

Transfection. A transfection kit was purchased from Foregene Co., Ltd. (TransEasy ${ }^{\mathrm{TM}}$ ). Ad-siRNA-Sirt3 was constructed by Sangon Biotech (Shanghai) Co., Ltd. In the 6-well plate used to culture $1 \times 10^{6}$ myocardial cells, Ad-siRNA-Sirt3 virus (1x10 $\mathrm{pfu} / \mathrm{virus})$ was used to infect the cells [multiplicity of infection $(\mathrm{MOI})=100]$. The cells were collected $24 \mathrm{~h}$ after infection. Subsequent to lysis by the protein lysate, the protein was extracted. A total of $50 \mu \mathrm{g}$ protein of each group was detected by western blot analysis. Whether the recombination
A
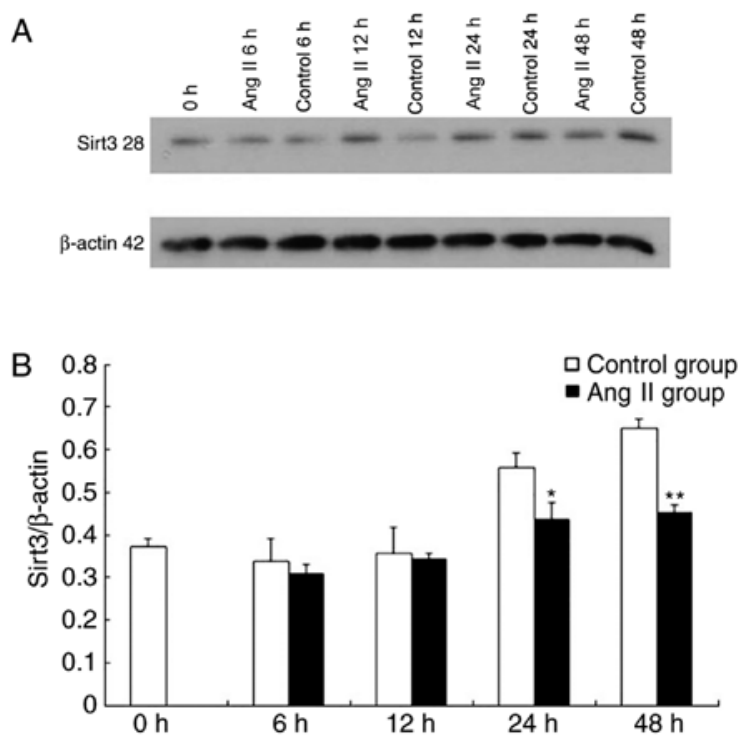

Figure 1. Influence of Ang II on Sirt3 protein expression at different time-points. (A) Shows the representative western blot of Sirt3 protein expression at 4 different time points in the control group and AngII group. (B) Sirt $3 / \beta$-actin ratio figure at 4 different time points. $n=4 .{ }^{*} \mathrm{P}<0.05,{ }^{* *} \mathrm{P}<0.01$ vs. respective control. Ang II, angiotensin II; Sirt3, sirtuin 3.

of adenovirus Ad-siRNA-Sirt3 was successful was determined utilizing the specific Sirt3 antibody and according to the expression alteration of the Sirt3 protein in each group.

The primary cultured myocardial cells of rats were used. The recombinant adenovirus vector Ad-siRNA-Sirt3 was used to infect the myocardial cells, and Ad-GFP and Ad-siRNA served as the control. The virus solution diluted into suitable titres was added to achieve the corresponding MOI (optimal MOI was $50 \mu \mathrm{M}$, according to the preliminary experiment; data not shown). After $6 \mathrm{~h}$ of culture at $37^{\circ} \mathrm{C}$, the virus solution was left, and DMEM culture medium containing a small amount of FCS was added for an additional $12 \mathrm{~h}$ of culture. After $48 \mathrm{~h}$ of treatment with $20 \mu \mathrm{M}$ Ang II, the mRNA expression of myocardial hypertrophy markers, atrial natriuretic peptide (ANP), brain natriuretic peptide (BNP), MCAD (a key enzyme of energy metabolism) and pyruvate kinase (PK), and the protein expression of Sirt3, LC3 and Beclin1, were detected.

Statistical analysis. The SPSS 15.0 (SPSS, Inc.) statistical package was used for the statistical analysis. The data of each group are presented as the mean \pm standard deviation. The differences between the groups and the differences between the time-points were analyzed by one-way analysis of variance, with the least significant difference method as the post hoc test. $\mathrm{P}<0.05$ was considered to indicate a statistically significant difference. All experiments were repeated three times.

\section{Results}

Sirt3 expression is decreased in Ang II-induced cardiomyocyte hypertrophy. The alterations in the expression of Sirt3 were observed in Ang II-induced cardiomyocyte hypertrophy to assess the role of Sirt3. The cardiomyocytes were treated with Ang II in vitro, and the expressions of Sirt3 were detected at 0 , $6,12,24$ and $48 \mathrm{~h}$. The results demonstrated that compared with 
the control group, the Sirt $3 / \beta$-actin ratio following treatment with Ang II decreased at different time-points (Fig. 1A and B). This suggested that Sirt3 is downregulated in Ang II-induced cardiomyocyte hypertrophy. The expression of LC3, which is a homologue of the autophagy-associated gene (GABA type A receptor associated protein like 2), in Ang II-induced cardiomyocyte hypertrophy decreased (data not shown). These results suggest that there is a potential association between the two (Fig. 1).

Res inhibits cardiomyocyte hypertrophy. The Sirt3 agonist Res was used to treat cardiomyocytes in Ang II-induced cardiomyocyte hypertrophy. It was observed that Res significantly inhibited cardiomyocyte hypertrophy in terms of cell surface area and the expression of hypertrophy-associated genes compared with the Ang II group (Fig. 2; P<0.05).

Res upregulates the expression of Sirt3, LC3 and Beclin1, and increases the energy metabolism of cardiomyocytes. The effects of Res on Sirt3 and autophagy were observed in Ang II-induced cardiomyocyte hypertrophy and the potential association between the two was assessed. The results demonstrated that in Ang II-induced cardiomyocyte hypertrophy, Res upregulated the expressions of Sirt3, autophagy-associated protein LC3 and Beclin1, which counteracted the effect of Ang II to a certain extent (Fig. 3A-C). With regard to the energy metabolism, Res increased the mRNA expression of MCAD while inhibiting the mRNA expression of PK (Fig. 3D and E).

Silencing Sirt3 reverses the effects of Res. As shown in Fig. 4, the effect of siRNA-mediated silencing of Sirt3 on cardiomyocytes and autophagy in Ang II-induced cardiomyocyte hypertrophy was observed. Following silencing, the expressions of Sirt3, LC3 and Beclin1 decreased; whereas, the mRNA expression of ANP and BNP increased. This effect counteracted the Res-induced inhibition on cardiomyocyte hypertrophy, increased the mRNA expression of PK and decreased the mRNA expression of MCAD. Consequently, the effect of Res on the energy metabolism in cardiomyocytes was reversed. These results demonstrated that the siRNA-mediated silencing of Sirt3 counteracted the promoting effect of Res on autophagy and its inhibitory effect on cardiomyocyte hypertrophy. From another perspective, it was confirmed that the Sirt3-autophagy signaling pathway served a role in cardiomyocyte hypertrophy.

\section{Discussion}

Hypertension is an important global health problem, causing myocardial cell hypertrophy (29). At present, inhibiting myocardial cell hypertrophy may block the signaling pathway of protein synthesis or promote the signal pathway of proteolysis (30). The present study examined the association between Sirt3 and autophagy in Ang II-induced myocardial cell hypertrophy, and assessed whether Sirt 3 affected myocardial cell hypertrophy and energy metabolism through autophagy.

Belonging to the NAD-dependent HDAC family, Sirt3 is primarily distributed in the mitochondria (31). A previous study identified that Sirt3 additionally exists in the cytoplasm and nuclei (32). The expression of Sirt3 is increased in organs with high metabolic activity, including brain tissue, the heart, liver and kidneys (33). Exercise, hunger, cold and oxidative stress may activate the expression of Sirt3 (34). Sirt 3 expression was significantly decreased in people with a high fat diet and those taking metformin, as well as in tumor cells (35-37). Sirt3 is involved in the regulation of approximately all cell metabolism-associated signaling pathways, including reactive oxygen species scavenging (38), tricarboxylic acid cycle (39-43), fatty acid oxidation $(34,44,45)$, ketogenesis $(46,47)$, protein synthesis $(48,49)$, cell growth and apoptosis (50-54). Sirt3 maintains the normal vital activities of the human body through multisystem and multi-link regulation, energy metabolism balance, anti-oxidative stress and the cell cycle (38). A number of previous studies demonstrated that Sirt3 may resist myocardial hypertrophy and heart failure through regulation of energy metabolism and reduction of oxidative stress. Sundaresan et al (10) observed that Sirt3 knockout mice demonstrated alterations of myocardial hypertrophy and myocardial fibrosis. Pillai et al (11) identified that myocardial hypertrophy may be inhibited by activating the Sirt3-LKB1-AMPK pathway. Chen et al (55) observed that Sirt3 may reduce myocardial fibrosis and improve the myocardial contraction through the transforming growth factor (TGF)- $\beta /$ mothers against decapentaplegic homolog 3 pathway. Furthermore, another previous study demonstrated that Sirt3 gene knockout aggravates the lipid deposition of the heart and suggests that abnormal energy metabolism may promote myocardial hypertrophy (56).

With respect to myocardial hypertrophy, autophagy may regulate the scavenging of cells, and maintain the mechanical function of the myocardium and the quality of the ventricle (55). Nakai et al (15) observed that autophagy related 5 (Atg5) gene knockout rats are more liable to ventricular hypertrophy, ventricular dilatation and abnormal contraction. Ucar et al (57) identified that miRNA-212/132 knockout mice have upregulated autophagy and significantly decreased myocardial hypertrophy. Another previous study suggested that the heart volume caused by myocardial hypertrophy is reduced by FoxO3 through upregulation of autophagy (58). Laurent et al (59) observed that exchanger 1 may activate the autophagy through calcium/calmodulin-dependent protein kinase kinase $\beta / \mathrm{AMPK}$; however, the downregulation of 3-methlyadenine and Atg5 (blockers of autophagy) may accelerate myocardial hypertrophy. It was suggested that the increase of autophagy is a reaction that balances cell hypertrophy to protect the myocardial cells.

Accumulating evidence suggests that excessive autophagy may accelerate myocardial cell death and aggravate heart failure. Zhu et al (60) observed that Beclin1 heterozygote $\left(\right.$ Beclin $\left.1^{+/}\right)$mice with downregulated autophagy demonstrated significantly improved ventricular remodeling and heart failure. Kostin et al (61) identified in heart failure subjects that moribund myocardial cells demonstrated significantly increased autophagy. Rawat et al (62) identified that increased active oxygen and abnormal energy metabolism may upregulate autophagy and accelerate the development of myocardial hypertrophy and diastolic heart failure. Therefore, a number of researchers suggested that cell death caused by autophagy may be the immediate cause of heart failure. Therefore, in the process of myocardial hypertrophy, moderate autophagy may 

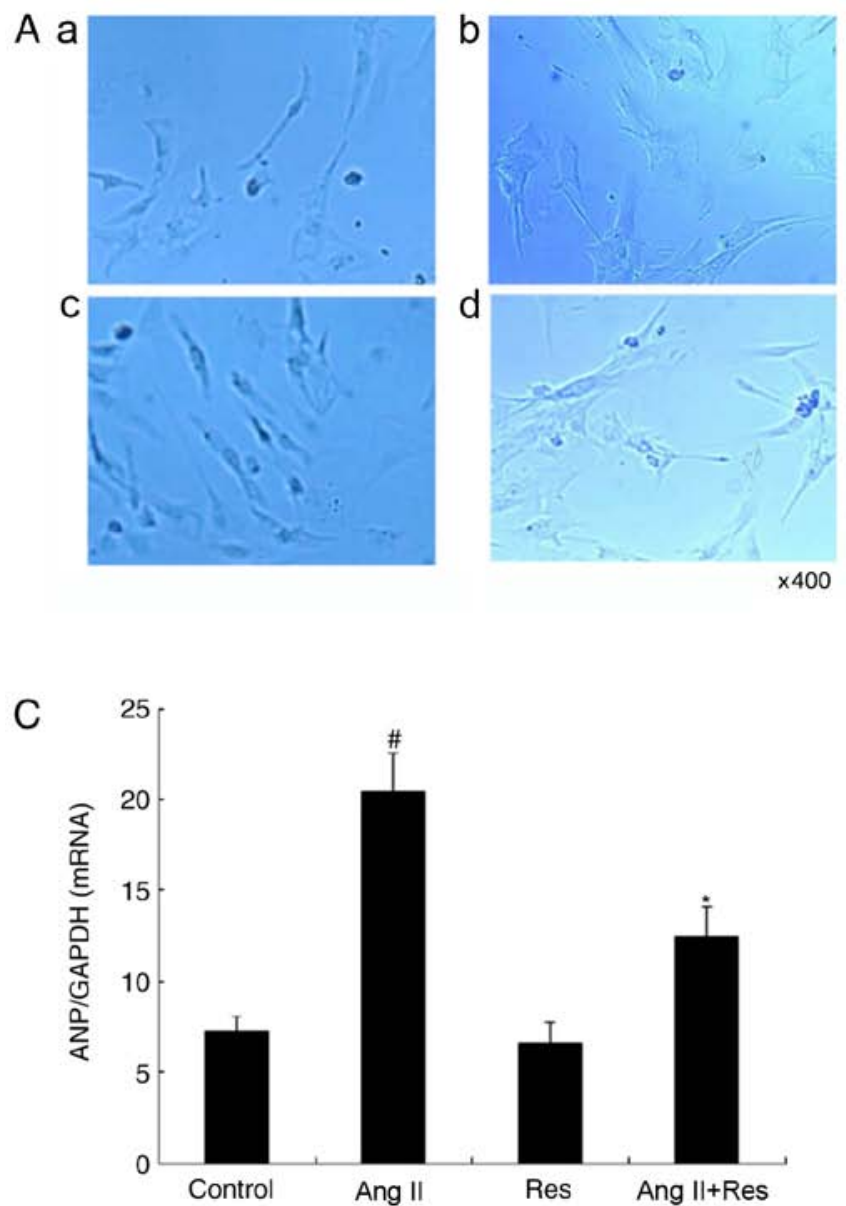
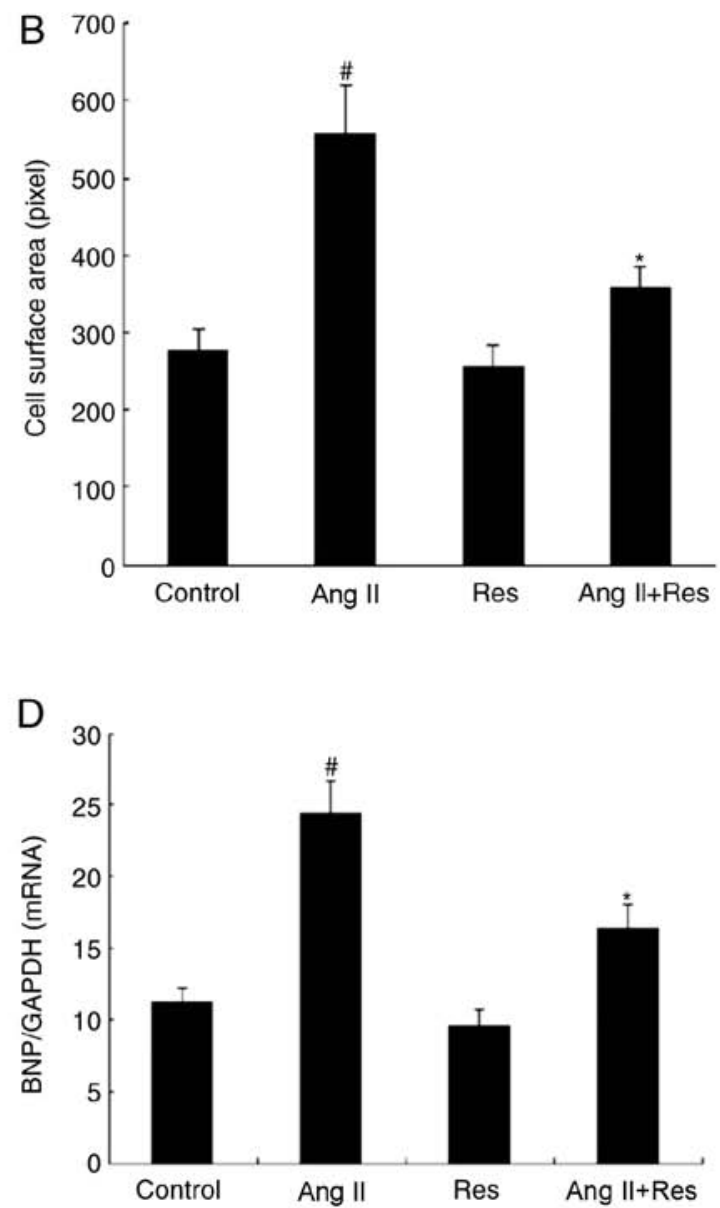

Figure 2. Influence of Res on protein expression in different treatment groups. (A) Influence of Res on the morphology of Ang II-induced hypertrophic myocardial cells. (Aa) Control group; (Ab) Ang II (20 $\mu \mathrm{M})$; (Ac) Res (50 $\mu \mathrm{M})$; and (Ad) initially treated with Res (50 $\mu \mathrm{M})$ and subsequently with Ang II (20 $\mu \mathrm{M})$ 30 min later. (B) Influence of Res on the area of Ang II-induced hypertrophic myocardial cells. (C) Influence of Res on the ANP mRNA in the Ang II-induced hypertrophic myocardial cells. (D) Influence of Res on the BNP mRNA in the Ang II-induced hypertrophic myocardial cells. $\mathrm{n}=4$. ${ }^{\#} \mathrm{P}<0.01$ vs. respective control; "P<0.05 vs. respective Ang II. Res, resveratrol; Ang II, angiotensin II; ANP, atrial natriuretic peptide; BNP, brain natriuretic peptide.

protect the myocardial cells; whereas, excessive autophagy will lead to the death of myocardial cells and the progression of heart failure (63). Autophagy exhibits different effects under the stimulation of different conditions and factors.

The present study examined the role of Sirt3-autophagy in myocardial hypertrophy. In a previous study, it was observed that in the process of Ang II-induced myocardial cell hypertrophy, the activity of autophagy decreased and the expression of the LC3 protein decreased significantly (16). It was additionally observed that a similar trend of downregulation of the Sirt3 protein was present in the identical cell model, which may be influenced by the NAD/reduced NAD ratio (16). To clarify the association between Sirt3 and autophagy, the present study used Res, which is an agonist of Sirt3, to intervene. It was identified that Res may increase the expression of Sirt3 and autophagy proteins, and inhibit the expression of ANP and BNP (myocardial cell hypertrophy factors), which suggested that Res may resist myocardial hypertrophy through activation of Sirt3 to induce autophagy. To further verify the observations, the present study combined adenovirus transfected siRNA-Sirt3 with Res intervention. It was observed that the expression of autophagy proteins was significantly decreased and the expression of ANP and BNP (myocardial cell hypertrophy factors) was significantly increased, which suggests that Sirt3 inhibited retinal neovascularization by regulating the migration-, neovascularization- and autophagy-associated factors expression (64). Sirt3 exerts protective effects in response to various damage factors involved in endothelial dysfunction, including Ang II, TGF- $\beta$ and high glucose (65). The reduced microvascular formation and VEGF expression in cardiac tissue was accompanied by a loss of mitochondrial Sirt3 during Ang II-induced cardiac remodeling (65).

In the present study, the expression of MCAD and PK was detected, and it was identified that the expression of MCAD was significantly decreased; however, the expression of PK was significantly increased in hypertrophic myocardial cells, which is consistent with a previous study (2). These results suggest that the energy utilization method of myocardial cells is altered and the efficiency of energy utilization decreases. Res intervention may reverse the alteration of MCAD and PK. However, the effect of Res decreases significantly following the combination of siRNA-Sirt3 and Res intervention. This result suggests that Sirt3 may promote fatty acid oxidation in myocardial cells, reduce the anaerobic glycolysis of glucose, lower the toxic effect of the intermediate product of glycolysis on myocardial cells and protect the heart accordingly (56).

The previous studies and the present results suggest that Sirt 3 activation may improve autophagy and inhibit myocardial 
A
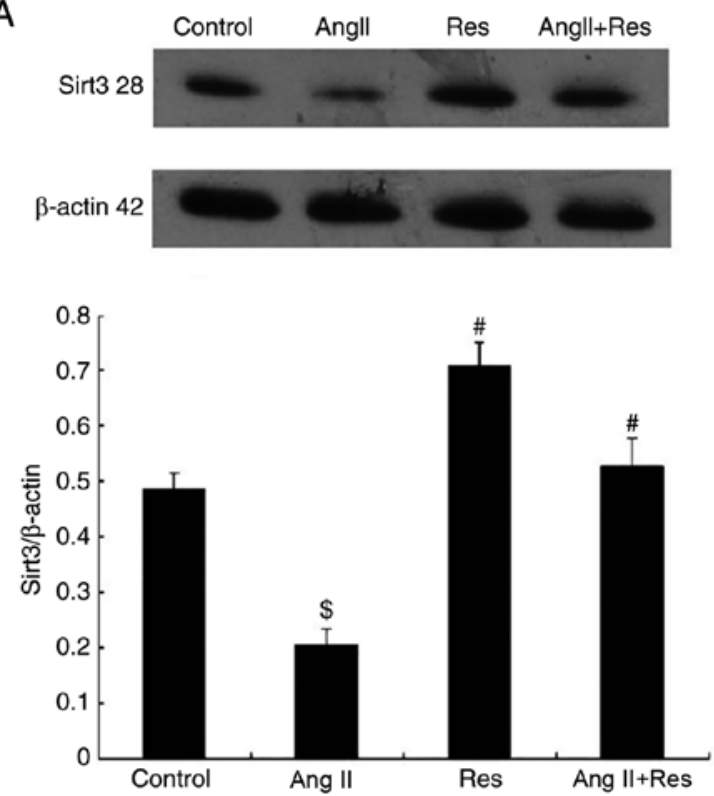

B

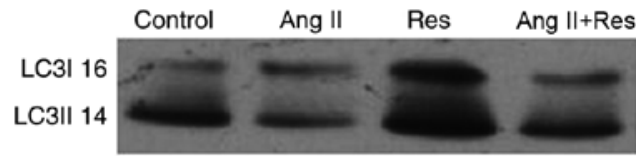

GAPDH 35

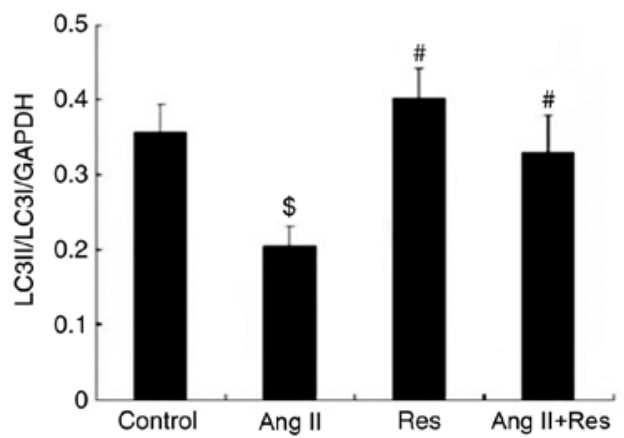

C

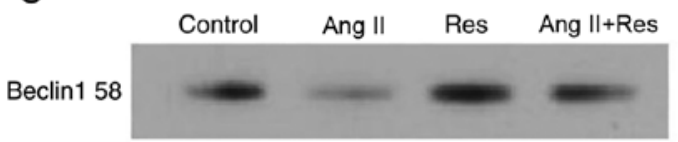

GAPDH 35

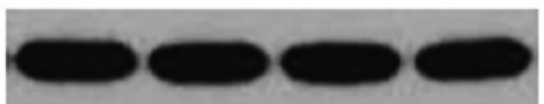

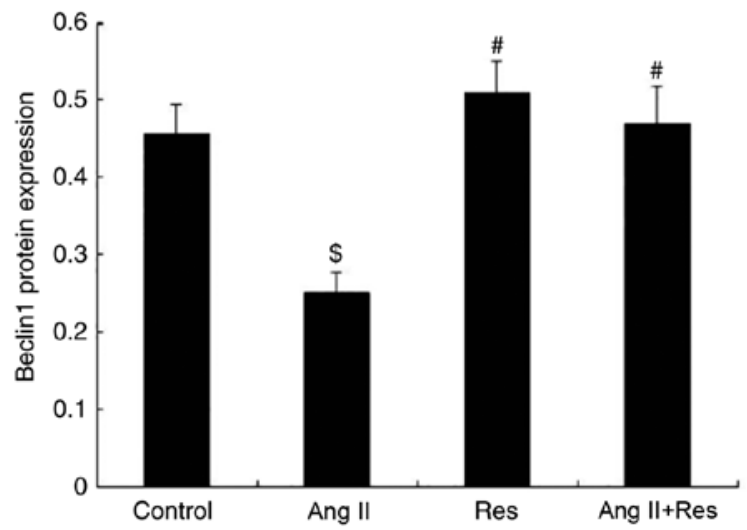

E

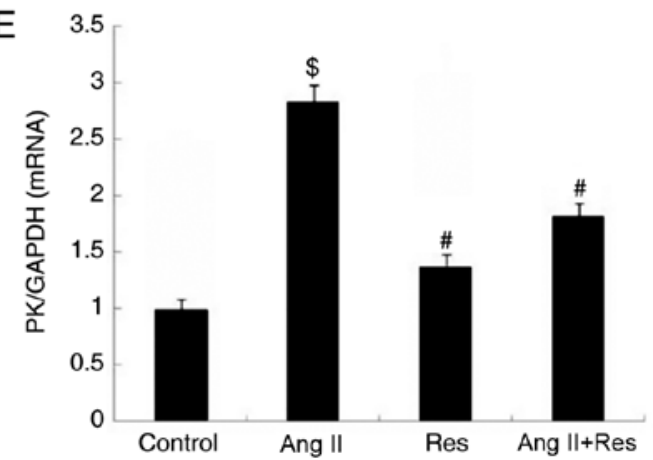

Figure 3. Influence of Res on protein expression in hypertrophic myocardial cells. (A) Influence of Res on the Sirt3 protein expression in the hypertrophic myocardial cells. (B) Influence of Res on the LC3 protein expression in the hypertrophic myocardial cells. (C) Influence of Res on the Beclin1 protein expression in the hypertrophic myocardial cells. (D) Influence of Res on the MCAD mRNA expression in the hypertrophic myocardial cells. (E) Influence of Res on PK mRNA in the hypertrophic myocardial cells. $n=4 .{ }^{\$} \mathrm{P}<0.01$ vs. the control group; ${ }^{\prime} \mathrm{P}<0.01$ vs. the Ang II group. Res, resveratrol; Sirt3, sirtuin 3; LC3, light chain 3; MCAD, medium-chain acyl-CoA dehydrogenase; PK, pyruvate kinase.

hypertrophy. In addition, Sirt3 protects the cell by reversing the abnormal energy metabolism caused by Ang II. As Sirt3 is vital in regulating cellular energy metabolism, cell growth and apoptosis, an increasing number of studies have identified that Sirt3 is closely associated with cardiovascular diseases, and Sirt3 may become a novel target for the treatment of cardiovascular diseases. Sirt3 is highly valuable for scientific research with strong potential for clinical application. The present study demonstrated that Sirt 3 may inhibit myocardial cell hypertrophy by regulating autophagy; however, further 

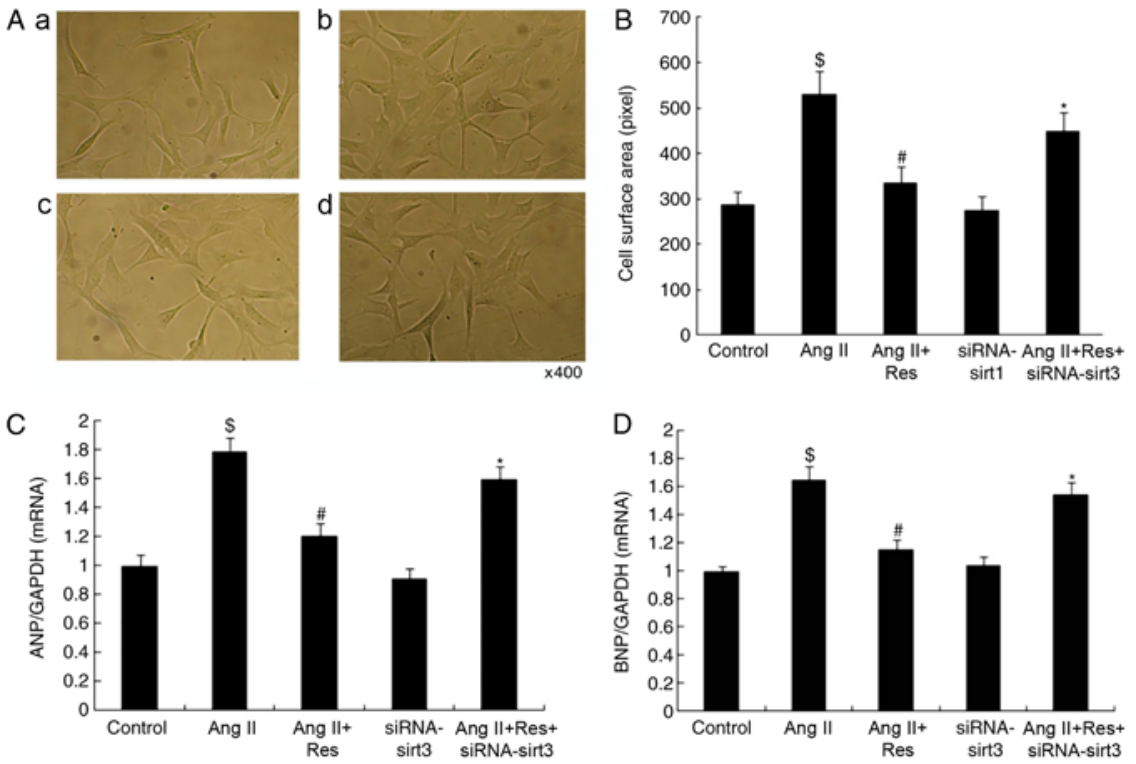

$\mathrm{E}$
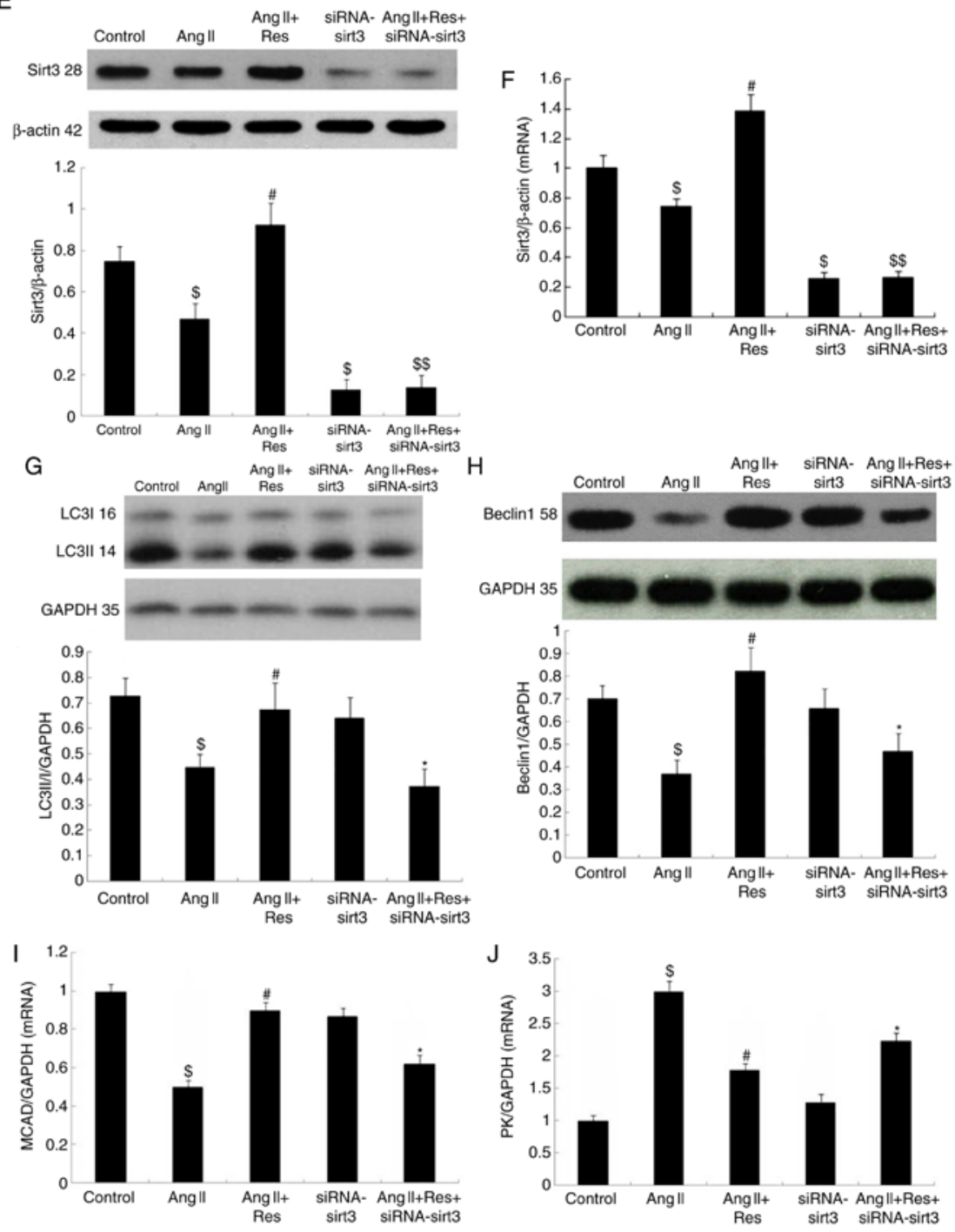

Figure 4. Influence of siRNA-Sirt3 in different treatment group. (A) Influence of siRNA-Sirt3 on the morphology of Ang II-induced hypertrophic myocardial cells. (Aa) Control group; (Ab) Ang II $(20 \mu \mathrm{M})$; (Ac) Res $(50 \mu \mathrm{M})$; and (Ad) initially treated firstly with Res $(50 \mu \mathrm{M})$ and subsequently with Ang II (20 $\mu \mathrm{M})$ 30 min later. (B) Influence of siRNA-Sirt3 on the area of Ang II-induced hypertrophic myocardial cells. (C) Influence of siRNA-Sirt3 on (C) ANP and (D) BNP mRNA in the Ang II-induced hypertrophic myocardial cells. Influence of siRNA-Sirt3 on Sirt3 (E) protein and (F) mRNA in the Ang II-induced hypertrophic myocardial cells. Influence of siRNA-Sirt3 on (G) LC3 and (H) Beclin1 protein expression in the Ang II-induced hypertrophic myocardial cells. Influence of siRNA-Sirt3 on (I) MCAD and (J) PK mRNA expression in the Ang II-induced hypertrophic myocardial cells. $n=4 .{ }^{\$} \mathrm{P}<0.05$, ${ }^{\$ \$} \mathrm{P}<0.01 \mathrm{vs}$. the control group; ${ }^{*} \mathrm{P}<0.01$ vs. the Ang II group; ${ }^{*} \mathrm{P}<0.05$ vs. the Ang II+Res group. siRNA, small interfering RNA; Sirt3, sirtuin 3; Ang II, angiotensin II; Res, resveratrol; ANP, atrial natriuretic peptide; BNP, brain natriuretic peptide; MCAD, medium-chain acyl-CoA dehydrogenase; PK, pyruvate kinase. 
investigations are required to determine the specific molecular mechanism of Sirt3-mediated regulation of autophagy.

\section{Acknowledgements}

Not applicable.

\section{Funding}

The present study was supported by a grant from the Guangdong Medical Research Fund (Guangzhou, China, grant no. B2012249).

\section{Availability of data and materials}

The data generated in the present study are available from the corresponding author upon reasonable request.

\section{Authors' contributions}

HNW and JLL designed the study and performed the experiments. TX and HQY performed the statistical analysis, GHC and $\mathrm{JH}$ were involved in designing the study, drafting the manuscript and conducted important revisions to the manuscript. All authors read and approved the final version of the manuscript.

\section{Ethics approval and consent to participate}

The present study was approved by the Medical Ethics Committee of Shantou University (Shantou, China).

\section{Patient consent for publication}

Not applicable.

\section{Competing interests}

The authors declare that they have no competing interests.

\section{References}

1. Vanberg P and Atar D: Androgenic anabolic steroid abuse and the cardiovascular system. Handb Exp Pharmacol 195: 411-457, 2010

2. Ruppert M, Korkmaz-Icöz S, Loganathan S, Jiang W, Lehmann L, Oláh A, Sayour AA, Barta BA, Merkely B, Karck M, et al: Pressure-volume analysis reveals characteristic sex-related differences in cardiac function in a rat model of aortic banding-induced myocardial hypertrophy. Am J Physiol Heart Circ Physiol 315: H502-H511, 2018.

3. Sugden PH and Clerk A: Cellular mechanisms of cardiac hypertrophy. J Mol Med (Berl) 76: 725-746, 1998.

4. den Besten G, van Eunen K, Groen AK, Venema K, Reijngoud DJ and Bakker BM: The role of short-chain fatty acids in the interplay between diet, gut microbiota, and host energy metabolism. J Lipid Res 54: 2325-2340, 2013.

5. Chatham JC and Young ME: Metabolic remodeling in the hypertrophic heart: Fuel for thought. Circ Res 111: 666-668, 2012.

6. Shen W, Asai K, Uechi M, Mathier MA, Shannon RP, Vatner SF and Ingwall JS: Progressive loss of myocardial ATP due to a loss of total purines during the development of heart failure in dogs: A compensatory role for the parallel loss of creatine. Circulation 100: 2113-2118, 1999.

7. Ingwall JS: Energy metabolism in heart failure and remodelling. Cardiovasc Res 81: 412-419, 2009.

8. Blander G and Guarente L: The Sir2 family of protein deacetylases. Annu Rev Biochem 73: 417-435, 2004.
9. Giralt A and Villarroya F: SIRT3, a pivotal actor in mitochondrial functions: Metabolism, cell death and aging. Biochem J 444: 1-10, 2012.

10. Sundaresan NR, Gupta M, Kim G, Rajamohan SB, Isbatan A and Gupta MP: Sirt3 blocks the cardiac hypertrophic response by augmenting Foxo3a-dependent antioxidant defense mechanisms in mice. J Clin Invest 119: 2758-2771, 2009.

11. Pillai VB, Sundaresan NR, Kim G, Gupta M, Rajamohan SB, Pillai JB, Samant S, Ravindra PV, Isbatan A and Gupta MP: Exogenous NAD blocks cardiac hypertrophic response via activation of the SIRT3-LKB1-AMP-activated kinase pathway. J Biol Chem 285: 3133-3144, 2010.

12. Yue Z, Ma Y, You J, Li Z, Ding Y, He P, Lu X, Jiang J, Chen S and Liu P: NMNAT3 is involved in the protective effect of SIRT3 in Ang II-induced cardiac hypertrophy. Exp Cell Res 347: 261-273, 2016.

13. Shintani T and Klionky DJ: Autophagy in health and disease: a double-edged sword. Science 306: 990-995, 2004.

14. Dämmrich J and Pfeifer U: Cardiac hypertrophy in rats after supravalvular aortic constriction. II. Inhibition of cellular autophagy in hypertrophying cardiomyocytes. Virchows Arch B Cell Pathol Incl Mol Pathol 43: 287-307, 1983.

15. Nakai A, Yamaguchi O, Takeda T, Higuchi Y, Hikoso S, Taniike M, Omiya S, Mizote I, Matsumura Y, Asahi M, et al: The role of autophagy in cardiomyocytes in the basal state and in response to hemodynamic stress. Nat Med 13: 619-624, 2007.

16. Wang HN, Liu C, Li YH and Chen Z, Sun G, Xue RC and Dong YG: Role of autophagy in myocardial hypertrophy induced by angiotensin II. J Zhongshan Univ 33: 440-443, 2014.

17. Hindle AG, Grabek KR, Epperson LE, Karimpour-Fard A and Martin SL: Metabolic changes associated with the long winter fast dominate the liver proteome in 13-lined ground squirrels. Physiol Genomics 46: 348-361, 2014.

18. Pi H, Xu S, Reiter RJ, Guo P, Zhang L, Li Y, Li M, Cao Z, Tian L, Xie J, et al: SIRT3-SOD2-mROS-dependent autophagy in cadmium-induced hepatotoxicity and salvage by melatonin. Autophagy 11: 1037-1051, 2015.

19. Tong W, Ju L, Qiu M, Xie Q, Cheng Y, Shen W, Sun W, Wang W and Tian J: Liraglutide ameliorate non-alcohol fatty liver disease by enhancing mitochondrial architecture and promoting autophagy through SIRT1/SIRT3-FOXO3a pathway. Hepatol Res 46: 933-943, 2016.

20. Auburger G, Gispert S and Jendrach M: Mitochondrial acetylation and genetic models of Parkinson's disease. Prog Mol Biol Transl Sci 127: 155-182, 2014.

21. Marques-Aleixo I, Santos-Alves E, Balça MM, Moreira PI, Oliveira PJ, Magalhães J and Ascensão A: Physical exercise mitigates doxorubicin-induced brain cortex and cerebellum mitochondrial alterations and cellular quality control signaling. Mitochondrion 26: 43-57, 2016.

22. Fang Y, Wang J, XuL,Cao Y, Xu F, Yan L, Nie M, Yuan N,Zhang S, Zhao R, et al: Autophagy maintains ubiquitination-proteasomal degradation of Sirt3 to limit oxidative stress in K562 leukemia cells. Oncotarget 7: 35692-35702, 2016.

23. Qiao A, Wang K, Yuan Y, Guan Y, Ren X, Li L, Chen X, Li F, Chen AF, Zhou J, et al: Sirt3-mediated mitophagy protects tumor cells against apoptosis under hypoxia. Oncotarget 7: 43390-43400, 2016.

24. Shi L, Zhang T, Zhou Y, Zeng X, Ran L, Zhang Q, Zhu J and Mi M: Dihydromyricetin improves skeletal muscle insulin sensitivity by inducing autophagy via the AMPK-PGC-1 $\alpha-S i r t 3$ signaling pathway. Endocrine 50: 378-389, 2015.

25. Mukherjee S, Ray D, Lekli I, Bak I, Tosaki A and Das DK: Effects of Longevinex (modified resveratrol) on cardioprotection and its mechanisms of action. Can J Physiol Pharmacol 88: 1017-1025, 2010.

26. Chen GQ, Liu C, Zhang CX, Meng RS, Chen BL, Xiong ZJ and Dong YG: The role of HO-1 in inhibiting cardiac hypertrophy by AMPK. Journal of Sun Yat-sen University, 31:614-618, 2010.

27. Yin R, Dong YG, Li HL and Liu D: Activated AMPK attenuates cardiac hypertrophy in rats through increasing myocardial fatty acid oxidation. Chin J Pathophysiol 23: 1258-1262, 2007.

28. Livak KJ and Schmittgen TD: Analysis of relative gene expression data using real-time quantitative PCR and the 2(- $\Delta \Delta \mathrm{C}(\mathrm{T}))$ method. Methods 25: 402-408, 2001.

29. Watson CJ, Horgan S, Neary R, Glezeva N, Tea I, Corrigan N, McDonald K, Ledwidge $M$ and Baugh J: Epigenetic therapy for the treatment of hypertension-induced cardiac hypertrophy and fibrosis. J Cardiovasc Pharmacol Ther 21: 127-137, 2016.

30. Lu L, Wu D, Li L and Chen L: Apelin/APJ system: A bifunctional target for cardiac hypertrophy. Int J Cardiol 230: 164-170, 2017. 
31. Cooper HM and Spelbrink JN: The human SIRT3 protein deacetylase is exclusively mitochondrial. Biochem $\mathrm{J}$ 411: 279-285, 2008

32. Scher MB, Vaquero A and Reinberg D: SirT3 is a nuclear $\mathrm{NAD}^{+}$-dependent histone deacetylase that translocates to the mitochondria upon cellular stress. Genes Dev 21: 920-928, 2007.

33. Jin L, Galonek H, Israelian K, Choy W, Morrison M, Xia Y, Wang X, Xu Y, Yang Y, Smith JJ, et al: Biochemical characterization, localization, and tissue distribution of the longer form of mouse SIRT3. Protein Sci 18: 514-525, 2009.

34. Shi T, Wang F, Stieren E and Tong Q: SIRT3, a mitochondrial sirtuin deacetylase, regulates mitochondrial function and thermogenesis in brown adipocytes. J Biol Chem 280: 13560-13567, 2005.

35. Li H, Feng Z, Wu W, Li J, Zhang J and Xia T: SIRT3 regulates cell proliferation and apoptosis related to energy metabolism in non-small cell lung cancer cells through deacetylation of NMNAT2. Int J Oncol 43: 1420-1430, 2013.

36. Bell EL, Emerling BM, Ricoult SJ and Guarente L: SirT3 suppresses hypoxia inducible factor $1 \alpha$ and tumor growth by inhibiting mitochondrial ROS production. Oncogene 30 2986-2996, 2011.

37. Jing E, Emanuelli B, Hirschey MD, Boucher J, Lee KY, Lombard D, Verdin EM and Kahn CR: Sirtuin-3 (Sirt3) regulates skeletal muscle metabolism and insulin signaling via altered mitochondrial oxidation and reactive oxygen species production. Proc Natl Acad Sci USA 108: 14608-14613, 2011.

38. Jacobs KM, Pennington JD, Bisht KS, Aykin-Burns N, Kim HS, Mishra M, Sun L, Nguyen P, Ahn BH, Leclerc J, et al: SIRT3 interacts with the daf-16 homolog FOXO3a in the mitochondria, as well as increases $\mathrm{FOXO}$ a dependent gene expression. Int J Biol Sci 4: 291-299, 2008

39. Fernandez-Marcos PJ, Jeninga EH, Canto C, Harach T, de Boer VC, Andreux P, Moullan N, Pirinen E, Yamamoto H, Houten SM, et al: Muscle or liver-specific Sirt3 deficiency induces hyperacetylation of mitochondrial proteins without affecting global metabolic homeostasis. Sci Rep 2: 425, 2012.

40. Finley LW, Haas W, Desquiret-Dumas V, Wallace DC, Procaccio V, Gygi SP and Haigis MC: Succinate dehydrogenase is a direct target of sirtuin 3 deacetylase activity. PLoS One 6: e23295, 2011.

41. Ahn BH, Kim HS, Song S, Lee IH, Liu J, Vassilopoulos A, Deng CX and Finkel T: A role for the mitochondrial deacetylase Sirt3 in regulating energy homeostasis. Proc Natl Acad Sci USA 105: 14447-14452, 2008.

42. Chen Y, Zhang J, Lin Y, Lei Q, Guan KL, Zhao S and Xiong Y: Tumour suppressor SIRT3 deacetylates and activates manganese superoxide dismutase to scavenge ROS. EMBO Rep 12: 534-541, 2011.

43. Finley LW, Carracedo A, Lee J, Souza A, Egia A, Zhang J, Teruya-Feldstein J, Moreira PI, Cardoso SM, Clish CB, et al SIRT3 opposes reprogramming of cancer cell metabolism through HIF1 $\alpha$ destabilization. Cancer Cell 19: 416-428, 2011.

44. Hallows WC, Yu W, Smith BC, Devries MK, Ellinger JJ, Someya S, Shortreed MR, Prolla T, Markley JL, Smith LM, et al: Sirt 3 promotes the urea cycle and fatty acid oxidation during dietary restriction. Mol Cell 41: 139-149, 2011.

45. Hirschey MD, Shimazu T, Goetzman E, Jing E, Schwer B, Lombard DB, Grueter CA, Harris C, Biddinger S, Ilkayeva OR, et al: SIRT3 regulates mitochondrial fatty-acid oxidation by reversible enzyme deacetylation. Nature 464: 121-125, 2010.

46. Hirschey MD, Shimazu T, Capra JA, Pollard KS and Verdin E: SIRT1 and SIRT3 deacetylate homologous substrates: AceCS1,2 and HMGCS1,2. Aging (Albany NY) 3: 635-642, 2011.

47. Shimazu T,Hirschey MD, Hua L, Dittenhafer-Reed KE, Schwer B, Lombard DB, Li Y, Bunkenborg J, Alt FW, Denu JM, et al: SIRT3 deacetylates mitochondrial 3-hydroxy-3-methylglutaryl CoA synthase 2 and regulates ketone body production. Cell Metab 12: 654-661, 2010.

48. Kendrick AA, Choudhury M, Rahman SM, McCurdy CE, Friederich M, Van Hove JL, Watson PA, Birdsey N, Bao J, Gius D, et al: Fatty liver is associated with reduced SIRT3 activity and mitochondrial protein hyperacetylation. Biochem $\mathrm{J} 433$ : 505-514, 2011.
49. Yang Y, Cimen H, Han MJ, Shi T, Deng JH, Koc H, Palacios OM, Montier L, Bai Y, Tong Q, et al: NAD ${ }^{+}$-dependent deacetylase SIRT3 regulates mitochondrial protein synthesis by deacetylation of the ribosomal protein MRPL10. J Biol Chem 285 7417-7429, 2010.

50. Hafner AV, Dai J, Gomes AP, Xiao CY, Palmeira CM, Rosenzweig A and Sinclair DA: Regulation of the mPTP by SIRT3-mediated deacetylation of CypD at lysine 166 suppresses age-related cardiac hypertrophy. Aging (Albany NY) 2: 914-923, 2010.

51. Iwahara T, Bonasio R, Narendra V and Reinberg D: SIRT3 functions in the nucleus in the control of stress-related gene expression. Mol Cell Biol 32: 5022-5034, 2012.

52. Allison SJ and Milner J: SIRT3 is pro-apoptotic and participates in distinct basal apoptotic pathways. Cell Cycle 6: 2669-2677, 2007.

53. Cooper HM, Huang JY, Verdin E and Spelbrink JN: A new splice variant of the mouse SIRT3 gene encodes the mitochondrial precursor protein. PLoS One 4: e4986, 2009.

54. Shulga N and Pastorino JG: Ethanol sensitizes mitochondria to the permeability transition by inhibiting deacetylation of cyclophilin-D mediated by sirtuin-3. J Cell Sci 123: 4117-4127, 2010.

55. Chen T, Li J, Liu J, Li N, Wang S, Liu H, Zeng M, Zhang Y and Bu P: Activation of SIRT3 by resveratrol ameliorates cardiac fibrosis and improves cardiac function via the TGF- $\beta / \mathrm{Smad} 3$ pathway. Am J Physiol Heart Circ Physiol 308: H424-H434, 2015.

56. Chen T, Liu J, Li N, Wang S, Liu H, Li J, Zhang Y and Bu P: Mouse SIRT3 attenuates hypertrophy-related lipid accumulation in the heart through the deacetylation of LCAD. PLoS One 10: e0118909, 2015.

57. Ucar A, Gupta SK, Fiedler J, Erikci E, Kardasinski M, Batkai S, Dangwal S, Kumarswamy R, Bang C, Holzmann A, et al: The miRNA-212/132 family regulates both cardiac hypertrophy and cardiomyocyte autophagy. Nat Commun 3: 1078, 2012.

58. Wang $\mathrm{X}$ and $\mathrm{Su} \mathrm{H}$ : FoxO3 hastens autophagy and shrinks the heart but does not curtail pathological hypertrophy in adult mice. Cardiovase Res 91: 561-562,2011.

59. Laurent AC, Bisserier M, Lucas A, Tortosa F, Roumieux M, De Régibus A, Swiader A, Sainte-Marie Y, Heymes C, Vindis C, et al: Exchange protein directly activated by cAMP 1 promotes autophagy during cardiomyocyte hypertrophy. Cardiovasc Res 105: 55-64, 2015.

60. Zhu H, Tannous P, Johnstone JL, Kong Y, Shelton JM, Richardson JA, Le V, Levine B, Rothermel BA and Hill JA: Cardiac autophagy is a maladaptive response to hemodynamic stress. J Clin Invest 117: 1782-1793, 2007.

61. Kostin S, Pool L, Elsässer A, Hein S, Drexler HC, Arnon E, Hayakawa Y, Zimmermann R, Bauer E, Klövekorn WP, et al: Myocytes die by multiple mechanisms in failing human hearts. Circ Res 92: 715-724, 2003

62. Rawat DK, Alzoubi A, Gupte R, Chettimada S, Watanabe M, Kahn AG, Okada T, McMurtry IF and Gupte SA: Increased reactive oxygen species, metabolic maladaptation, and autophagy contribute to pulmonary arterial hypertension-induced ventricular hypertrophy and diastolic heart failure. Hypertension 64: 1266-1274, 2014.

63. De Meyer GR, De Keulenaer GW and Martinet W: Role of autophagy in heart failure associated with aging. Heart Fail Rev 15: 423-430, 2010.

64. Mao XB, You ZP, Wu C and Huang J: Potential suppression of the high glucose and insulin-induced retinal neovascularization by Sirtuin 3 in the human retinal endothelial cells. Biochem Biophys Res Commun 482: 341-345, 2017.

65. Wei T, Huang G, Gao J, Huang C, Sun M, Wu J, Bu J and Shen W: Sirtuin 3 deficiency accelerates hypertensive cardiac remodeling by impairing angiogenesis. J Am Heart Assoc 6: e006114, 2017.

This work is licensed under a Creative Commons Attribution-NonCommercial-NoDerivatives 4.0 International (CC BY-NC-ND 4.0) License. 